\title{
Digital and Conventional Film-Based Non-Metric Cameras: A Comparison of Performance in Structural Deformation Surveys
}

\begin{abstract}
Abdullah S. Alsalman ${ }^{1}$
Abstract

This research work presents the results of an experiment concerned with a comparison of the performance of non-metric digital and non-metric film-based cameras for structural deformation surveys. An Olympus OM 10 non-metric camera and a Fujix DS100 still image digital camera were used to measure the amount of deformation of a model of a light-weight building when under load. For the sake of consistency, the same photogrammetric network design, control configuration and self -calibrating bundle adjustment were used for data evaluation. The results of the research show that digital nonmetric cameras are viable, and possibly accurate, tools for structural deformation studies .Taking into account the obvious advantages of ease of operation, relatively low cost and computer-amenability, the author appeals to surveyors and civil engineers to consider digital cameras in their respective deformation survey studies and practices.
\end{abstract}

ملخص

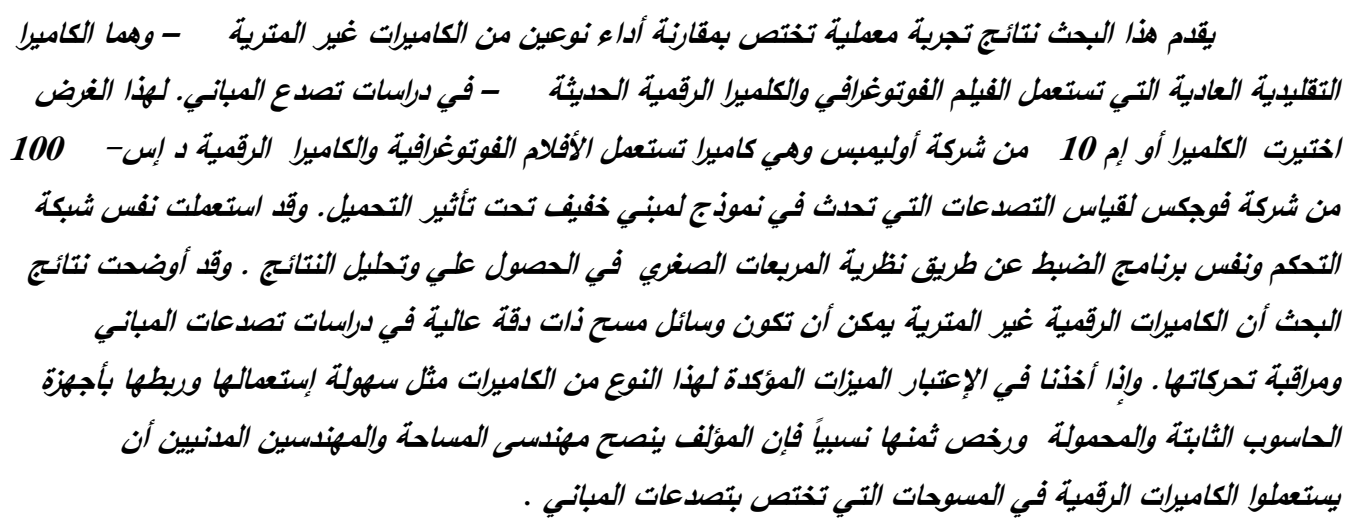

\footnotetext{
${ }^{1}$ Civil Engineering Department, College of Engineering, King Saud University
} 


\section{Introduction}

Non-conventional metric (close-range, terrestrial or non -topographic) photogram metric methods are now often used in deformation surveys in many engineering applications; especially those concerned with car industry, boiler surface monitoring, architectural analysis and model deformation studies. Generally, precise measurement of photo coordinates of discrete points on a model or a prototype are carried out in two or more epochs using a precise measuring instrument e.g. a comparator, a tablet digitizer or an on-screen method.

In the last ten to fifteen years, analytical close-range photogrammetry has reached a high level of accuracy which made it feasible and practical for a multitude of precision measurement engineering works .On the other hand, digital close-range photogrammetry has recently become potential rival for many applications. The main merits of digital close -range photogrammetry are ease and speed of operation of devices, accurate automated data capture and availability of efficient evaluation tools.

In fact, developments in digital photogram metric techniques have reached a high degree of maturity for a variety of applications (Wolf and Dewitt, 2000, Atkinson, 1996). One important such application is in structural deformation monitoring using test models subjected to a series of loads at different epochs.

In this article, the performance of a digital camera is compared to that of a non-metric film -based camera for deformation surveys. Model set-up, design of the network of points used in the experiment, data acquisition and evaluation are briefly discussed. Results of the test are outlined and analyzed and conclusions as regards advantages and use of digital close -range photogrammetry are presented.

\section{Model Preparation}

A structural model of a light weight roof was utilized to compare the performance of an off-the-self digital camera with that of a film-based nonmetric camera. The model used in the test comprises a wooden box, $90 \mathrm{~cm}$ long and $60 \mathrm{~cm}$ wide. The box is not rectangular, however. Along the longer sides, it is $15 \mathrm{~cm}$ high, and is $25 \mathrm{~cm}$ high on the shorter ones.

A wire mesh of $10 \times 15$ wires was made to span from opposite walls, thus simulating hanging and standing cables as used in structural design. The 
wires were separated by $5 \mathrm{~cm}$ in both directions, thus creating 150 intersection points. These are then used as "surveyed or monitored points" in order to study the deformations that take place in the surface roof.

From experience gained elsewhere e.g. (Fraser, 1992), with a white background, as is the case in this experiment, wires with black color were used in order to ease identification of the points and interpretation of the results. Also, for photo scales ranging from $1 / 100$ to $1 / 300$, wires with cross-sections having diameters of around 9 micrometers are appropriate for such kind of tests (Hanari, 1981, Ghosh, 1980).

\section{Coordination of object space control}

Fifteen control points with different elevations were constructed in the test model with 4 more to improve the geometric fidelity of the photogram metric network.

To improve set-up of the test, the shape, size and color of the control points had to be decided. Previous experience (e.g. Hanari , 1981) showed that either square or circular targets with black and white combination are most suitable for such cases. The size of these targets was chosen to be in the order of $1 \mathrm{~cm}$. This is believed to accommodate the range of photo scales to be used in the test.

Since the accuracy of the control points directly influences the photogrammetric results, a control survey of high accuracy was carried out using a high accuracy electronic mapping system consisting of a pair of Kern E2 one-second electronic theologies interfaced with a Pentium IV IBM personal computer. To provide a nominal scale for this measuring system, a Wild SBL invar sub tense bar (length known to an accuracy of 1/150000) was used (Fig. 1). 


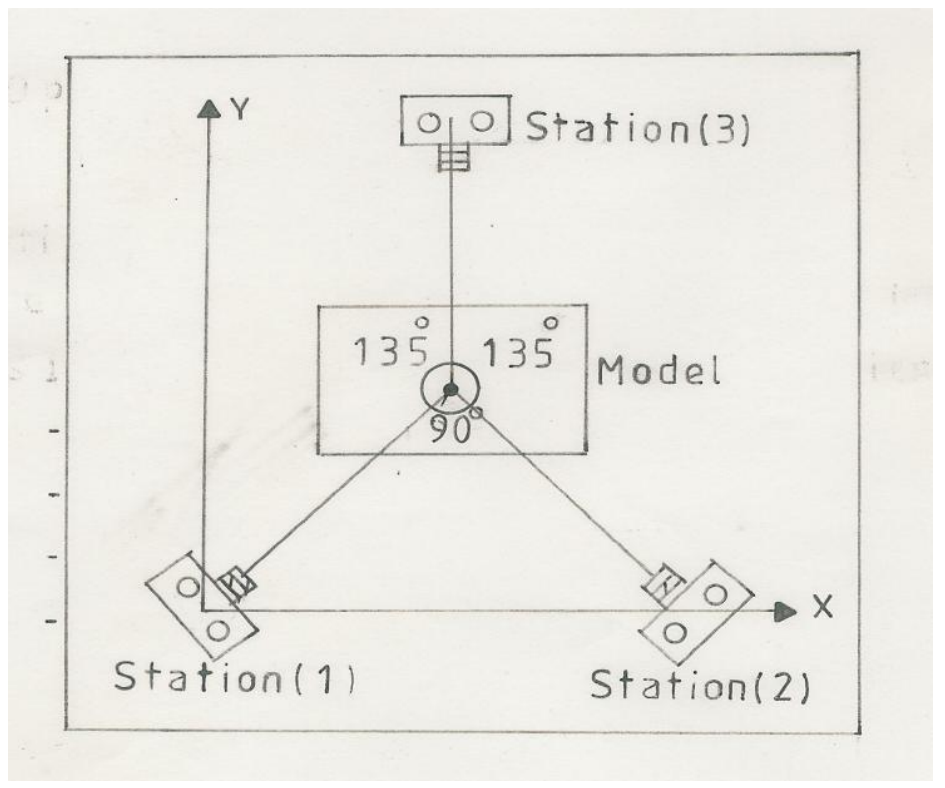

Fig (1): Control survey configuration

The observational procedure undertaken in this test consists of traditional intersection (i.e. horizontal directions and zenith distances observed for each point to be measured). The observed values are directly fed into the computer for further processing, i.e. manual booking was not necessary. After each measurement, a bundle adjustment computer program was carried out in order to obtain three dimensional coordinates for all test points as well as accuracy information.

All horizontal directions and zenith distances were observed in two sets .The average standard deviation for the final coordinates of the control points were $0.04 \mathrm{~m}, 0.03 \mathrm{~mm}$, and $0.02 \mathrm{~mm}$ in $\mathrm{x}, \mathrm{y}$, and $\mathrm{z}$ directions respectively.

\section{Photographic Set-up}

Usually, in deformation surveys terrestrial photos are taken from two suitable camera stations at two different epochs, the first before loading and the second one after the load has been applied. The two sets of photographic data are then processed and use to compute three-dimensional coordinates of all points of interest. These computed coordinates represent the roof surface in the 
two cases. The differences between the two sets of coordinates represent the deformation information caused by loading the roof surface.

For the purpose of establishing favorable geometric configuration, a photogram metric network with a three -station configuration was established to measure the deformation values. The photographic overlap for this system was $100 \%$ for each pair of convergent photographs (see Fig. 2).



Fig (2): Optimum Camera setup

The attention of the reader is brought to the fact that the image formats of the two cameras (the non-metric film-based camera and the digital camera) and their respective focal lengths are necessarily different. This gives rise to two photo exposure options. In the first one, the distance between the camera and the object is kept constant; and in this case, the two photographs will have different scales. In the second, the photo scale is kept constant while changing the camera-to-object distance. From a practical point of view, the first option was adopted.

The camera-to-object distance was chosen to be in the order of $2.5 \mathrm{~m}$. This value was chosen based on experience gained by other investigators (e.g. 
Abdelaziz, 1971, Fraser, 1992). This resulted in photo scale values of around $1 / 90$ for the film-based non-metric camera and 1/320 for the digital camera.

\section{Mensuration Equipment Used In the Test}

The film-based non-metric camera used in the test is the famous Olympus model OM-10 having the following characteristics:-

- $\quad$ Nominal focal length $=28 \mathrm{~mm}$.

- Size of photo format $=36 \mathrm{~mm} \times 24 \mathrm{~mm}$.

- $\quad$ Range of f-stop values $=2.8-22$.

- An adaptor for automatic exposure time.

The camera has eight artificial fiducial marks engraved in the photo plane for the purpose of an earlier project concerned with surveying an archeological site. The photographic film used for capturing the images was a Kodak Elite 400 diapositive color film. For the sake of maximizing the depth of field, an aperture size of $f / 22$ was utilized. This gives rise to a diaphragm opening in the vicinity of $1.3 \mathrm{~mm}$. The test was carried out indoors in a constant temperature and constant humidity environment. This is believed to be advantageous since it seems to minimize external influences on the experiment. Furthermore, the light conditions in the laboratory made it possible to set the exposure time to one second.

The other camera used in the test is a Fujix DS-100 still- memory card digital camera. It is a low- cost, off-the-shelf, ammeter-type digital camera .Generally; it is not specifically designed for any particular scientific or photogram metric tasks. In this respect, it can be considered as a non-metric digital camera since, like conventional non-metric film-based cameras, it has similar weak geometric characteristics. The main difference in this case is the recording medium.

The focal length of this camera varies between 8 and $30 \mathrm{~mm}$ for $3 \mathrm{x}$ power zoom lens having f-stop values ranging from 2 to 11 and exposure time settings ranging from $1 / 4$ to $1 / 750 \mathrm{sec}$. The picture element is a chargecoupled device (CCD) solid state image sensor. The frame size of the camera is $720 \times 488$ pixels with a pixel size of $8.5 \mu \mathrm{m} \times 9.7 \mu \mathrm{m}$. The storing medium is an Image Memory Card (IMC) with 8 mega byte storing capacity. In order to display the image stored in the card, it is first resampled on the computer using a memory card processor and an appropriate digital still camera system 
image handling software. The resampled image has dimensions of 640 x 488 pixels with a square pixel size of approximately $9.6 \mu \mathrm{m} \times 9.6 \mu \mathrm{m}$.

In order to obtain maximum angular field of view for image capturing, the minimum value of $8 \mathrm{~mm}$ for the focal length was used. From an earlier similar experience with this camera (Ali, 2003), the maximum value of f-stop $=11$ and a maximum exposure time of $\mathrm{t}=0.25$ second were chosen .

Three sets of data were available for analysis. These are:-

- Data obtained from film-based non-metric camera.

- Data obtained from the digital camera.

- Data acquired by scanning the hard-copy photos of the conventional film-based camera.

For the last type of data, a Canon Color laser Copier model CLC -200 was used for the purpose .The manufacturer of this device states that it has a resolution of $400 \mathrm{dpi}$ (dots (or pixels) per inch) and a graduation of 256 tones or levels per color (blue, green ,red). This copier can scan directly from prints or slides. The final pixel size was in the order of $9.6 \mu \mathrm{m} \times 9.6 \mu \mathrm{m}$.

The output image was chosen to have a format of $1.325 \mathrm{~cm} \times 1.000 \mathrm{~cm}$ which produced a frame of 13475 x 10170 pixels. The photo scale for the scanned images was calculated and was found to be in the order of 1:160 with a hypothetical focal length of $16 \mathrm{~mm}$

\section{Measurement of the photo coordinates}

Measurement of the six hardcopy images was undertaken using a Wild AC-1 analytical restitution system utilized in a monocomparator measurement mode. Each photo was measured three times. The average photo coordinates and their root-mean-square values were then computed. An average root-meansquare value was then calculated for each photo in order to give an indication on the precision of the measurement process .Table (1) illustrates the precision of the observations for both epochs. 
Digital and Conventional Film-Based Non-Metric Cameras...

Abdullah S. Alsalman

Table (1): Measurement Precision for the hardcopy Images.

\begin{tabular}{|c|c|c|c|c|}
\hline \multirow{2}{*}{ Station } & \multicolumn{2}{|c|}{ Before Deformation } & \multicolumn{2}{c|}{ After Deformation } \\
\cline { 2 - 5 } & $\mathrm{x}(\mu \mathrm{m})$ & $\mathrm{y}(\mu \mathrm{m})$ & $\mathrm{x}(\mu \mathrm{m})$ & $\mathrm{y}(\mu \mathrm{m})$ \\
\hline$(1)$ & 3.77 & 4.32 & 2.93 & 3.00 \\
\hline$(2)$ & 5.39 & 6.45 & 4.11 & 4.26 \\
\hline$(3)$ & 4.11 & 4.99 & 3.40 & 3.99 \\
\hline
\end{tabular}

For the two sets of digital data, an old first generation digital video plotter from Wild was used. This device has the advantages of low cost (compared to photogram metric workstations) and powerful photogram metric software. In addition, it can be attached to any PC without the need for a high storage hard drive device. Nevertheless, this digital plotter has an accuracy that matches the accuracy values of most mainstay analytical plotters.

Each digital image (original or scanned) was measured three times and the average coordinate values and root-mean-square errors were calculated. These are presented in Tables (2) and (3).

Table (2): Measurement Precision of Digital Images

\begin{tabular}{|l|l|l|l|l|l|l|l|l|}
\hline \multirow{3}{*}{ Station } & \multicolumn{5}{|c|}{ Before Deformation } & \multicolumn{3}{c|}{ After Deformation } \\
\cline { 2 - 10 } & \multicolumn{2}{|c|}{$\mathrm{x}$} & \multicolumn{2}{c|}{$\mathrm{y}$} & \multicolumn{2}{c|}{$\mathrm{x}$} & \multicolumn{2}{c|}{$\mathrm{y}$} \\
\cline { 2 - 10 } & $\mathrm{Pixel}$ & $\mu \mathrm{m}$ & Pixel & $\mu \mathrm{m}$ & Pixel & $\mu \mathrm{m}$ & Pixel & $\mu \mathrm{m}$ \\
\hline \multirow{2}{*}{$(1)$} & 0.07 & 0.90 & 0.10 & 1.01 & 0.04 & 0.50 & 0.06 & 0.55 \\
\hline & & & & & & & & \\
$(2)$ & 0.05 & 0.60 & 0.07 & 0.79 & 0.04 & 0.50 & 0.06 & 0.62 \\
\hline$(3)$ & 0.05 & 0.59 & 0.08 & 0.70 & 0.04 & 0.45 & 0.06 & 0.53 \\
\hline
\end{tabular}


مجلة العلوم الهندسية- العدد الرليع- 2009

Table (3) Measurement Precision of Scanned Photos:

\begin{tabular}{|l|l|l|l|l|l|l|l|l|}
\hline \multirow{2}{*}{ Station } & \multicolumn{5}{|c|}{ Before Deformation } & \multicolumn{5}{c|}{ After Deformation } \\
\cline { 2 - 10 } & \multicolumn{2}{|c|}{$\mathrm{x}$} & \multicolumn{2}{|c|}{$\mathrm{y}$} & \multicolumn{3}{c|}{$\mathrm{x}$} & \multicolumn{2}{c|}{$\mathrm{Y}$} \\
\cline { 2 - 10 } & Pixel & $\mu \mathrm{m}$ & Pixel & $\mu \mathrm{m}$ & Pixel & $\mu \mathrm{m}$ & Pixel & $\mu \mathrm{m}$ \\
\hline$(1)$ & 0.11 & 1.01 & 0.13 & 1.30 & 0.12 & 1.19 & 0.11 & 1.01 \\
\hline$(2)$ & 0.12 & 1.27 & 0.13 & 1.29 & 0.10 & 0.96 & 0.08 & 0.94 \\
\hline$(3)$ & 0.10 & 0.91 & 0.07 & 0.84 & 0.08 & 0.68 & 0.08 & 0.72 \\
\hline
\end{tabular}

\section{Data Evaluation}

For the evaluation of the results, it was decided to use an off-the-shelf self-calibrating bundle adjustment program in order to minimize the effects of some unknown systematic errors inherent in the digital and the film-based photographic data. A version of Program BUNDAJ developed by Prof. Ali of the University of Khartoum, Sudan, was made available to the author .The program has the following advantages:-

- No additional control information is needed for the calibration.

- No approximate values for the unknown parameters are required except the approximate value of the principal distance.

- the calibration is photo-type-independent , and

- It does not require presence of fiducial marks.

Input information to the program includes measured photo coordinates, control data, and the number of iterations required to solve for the unknown parameters. The output information consists of three-dimensional coordinate values for the unknown points in the object coordinate system, the camera calibration parameters and statistical information regarding the accuracy of the work.

Tables 4 and 5 present a comparison of the results pertinent to the three types of data as obtained from the bundle adjustment program. 
Table (4:( Accuracy of Photo Coordinates) in micrometers) for the Three Data Types after Using Bundle Adjustment.

\begin{tabular}{|c|c|c|c|c|}
\hline \multirow{2}{*}{ Project } & \multicolumn{2}{|c|}{$\begin{array}{c}\text { Before Deformation } \\
(\mu \mathrm{m})\end{array}$} & \multicolumn{2}{c|}{$\begin{array}{c}\text { After Deformation } \\
(\mu \mathrm{m})\end{array}$} \\
\cline { 2 - 5 } & rmse $\mathrm{x}$ & rmse y & 3.64 & rmse y \\
\hline Hard - copy & 5.01 & 5.39 & 2.59 & 3.60 \\
\hline Digital & 3.15 & 3.18 & 3.71 & 1.84 \\
\hline Scanned & 5.50 & 3.23 & & 2.11 \\
\hline
\end{tabular}

Table (5) Accuracy of Photo Coordinates of the Check Points )in micrometers) for the Three Data Types after Bundle Adjustment

\begin{tabular}{|c|c|c|c|c|c|c|}
\hline \multirow{2}{*}{ Project } & \multicolumn{3}{|c|}{$\begin{array}{c}\text { Before Deformation } \\
(\mathrm{mm})\end{array}$} & \multicolumn{3}{c|}{ After Deformation } \\
\cline { 2 - 8 } & $\mathrm{x}$ & $\mathrm{y}$ & $\mathrm{z}$ & $\mathrm{x}$ & $\mathrm{y}$ & $\mathrm{z}$ \\
\hline Hard - copy & 0.34 & 0.19 & 0.31 & 0.10 & 0.20 & 0.58 \\
\hline Digital & 0.12 & 0.02 & 0.18 & 0.26 & 0.38 & 0.48 \\
\hline Scanned & 0.50 & 0.32 & 0.53 & 0.47 & 0.77 & 0.36 \\
\hline
\end{tabular}

\section{Concluding Remarks}

Tables (4) and (5) are largely self-explanatory. However, it is possible to strengthen them with some additional remarks. A quick glance at these tables reveals that off-the-shelf ammeter digital cameras, as exemplified by the Fujix DS-100 used in this test, can give accuracy figures approaching those obtained by conventional film-based non-metric cameras. In fact, the results of the present study show a slightly higher accuracy in favor of the digital camera. This is especially true with the check points. Thus, accuracy values as small as 20 micrometers were obtained for the digital camera. The scanned version of the photos gave accuracy values markedly lower than the other two types of data .This can be attributed to the fact that commercial electronic copiers add 
extra geometric errors caused by the electronic copying process. This is a point evaluated by Ali (1998). The reader is advised to refer to that publication for more details on this. However, for most surveys of this nature, this range of accuracy is quite tolerable (Hanari, 1981, Ghosh, 1980). Thus, if the small format can tolerated, digital non-metric photogrammetric data can serve a useful purpose in monitoring structural deformations. This application can be extended to include surveys related to architecture, archeology, hydrographic laboratory modeling, metallurgy, car industry, fracture surveys in fine arts, dentistry, fetus and tumour studies in medicine, etc.

\section{References}

(1) Abdelaziz, Y.A. And Karara, H.M., .1971Direct Linear Transforms from Comparator Coordinates into Object-space Coordinates .In American Society of Photogrammetry Symposium on Close-range Photogrammetry,VA, pp.1-,18

(2) Ali, A.E., .2003Close-Range Photogrammetry in Bridge Movement Monitoring .Technical report no. TR-003-1, Ministry of Engineering Affairs, State of Khartoum, Sudan.

(3) Ali, A.E., 1998. Consequences of Enlaging Small-format Photographs with Commercial Copiers .Journal of King Saud University) engineering sciences)

(4) Atkinson, K.B. ed. 1996. Close-range Photogrammetry and Machine Vision. First Edition, Caithness, Scotland, Whittles Publishing,(386 pages) .

(5) Fraser, C.S., 1992. Photogrammetric Measurements to one Part per million .Photogrammetric Engineering and Remote Sensing, Vol. ,58 No. 3, pp.305-312.

(6) Ghosh, S.K., 1980. Photogrammetry for Police Use: Experience from Japan .Photogrammetric Engineering and Remote Sensing, Vol. ,46 No. ,3 pp. 329-338.

(7) Hanari, G,. 1981. Close -range Photogrammetry in Coal Mine Industry . M.Sc. Thesis (220 pages.( University of Glasgow, Scotland (Unpublished).

(8) Kenefick, J. F., 1977. Application of Photogrammetry in Ship-building . Photogrammetric Engineering and Remote Sensing, Vol. 43, no. ,9 pp 11691175 .

(9) Wolf, P.R. and Dewitt, B.A., 2000. Elements of Photogrammetry with GIS Applications in GIS. McGraw-Hill, New York, Third Edition , (608 pages). 